\title{
Mini-commentary on BJOG-20-0372.R2 Risk of preeclampsia in patients with maternal genetic predisposition to common medical conditions: a case-control study
}

\author{
Austin Ugwumadu ${ }^{1}$ \\ ${ }^{1}$ St Georges, University of London
}

September 11, 2020

The American obstetrician, Joseph DeLee, 'father of modern obstetrics', founded the Chicago Lying-in Hospital in 1931 with his vision for womens' health captured in a set of five stone plaques at the top of the cloister of the hospital's building. Four of the five stone plaques are engraved with the names of pioneering clinicians whose contributions to the field of obstetrics and gynaecology have been seminal: Jan Palfyn (16501730), for introducing the obstetric forceps in the 1720s, Hendrik Van Deventer (1651-1724), for discovering obstetric anatomical disorders, William Smellie (1697-1763), for improving the design of the forceps, and Eduardo Porro (1842 -1902), for introducing hysterectomy to stop postpartum haemorrhage. The fifth stone plaque, in the centre, which is still blank today, is reserved for the physician/scientist who discovers the cause and cure of preeclampsia according to legend.

That clusters of preeclamsia occur in family units prompted the question whether preecclampsia is an inherited disorder, and if so, what is the mode of inheritance? Familial clustering opened the possibility of deploying genome wide association studies (GWAS) for the identification of candidate genes and susceptibility loci for the development of preeclampsia and researchers have focused on this question since the discovery of DNA. However, till date, there has been no clearly defined causal relationship between a preeclampsia genotype and phenotype except for heterozygous women who are pregnant with long-chain hydroxyacyl-CoA dehydrogenase deficient fetus who have nearly $80 \%$ chance of developing HELLP syndrome or acute fatty liver of pregnancy. Defining the genetics and mode of inheritance of preeclampsia is challenged in part by the involvement of two genomes (maternal and fetal), and a wide spectrum of women who meet the diagnostic criteria according to the International Society for the Study of Hypertension in Pregnancy.

There is persuasive literature that coexisting maternal medical disorders such as diabetes, chronic hypertension, renal disease, autoimmune disease, antiphospholipid antibody syndrome and other maternal-fetal risk factors including obesity, dyslipidemia, nulliparity, previous/family history of preeclampsia, and multifetal gestation, increase the risk of preeclampsia. In this issue of the Journal, Gray and colleagues (BJOG 2020 xxxx) used single nucleotide polymorphisms (SNPs) for 21 distinct clinical traits for increased risk of preeclampsia within 7 categories (cardiovascular, inflammatory/ autoimmune, insulin resistance, liver, obesity, renal, and thrombophilia) from the European GWAS to test the hypothesis that women with genetic predisposition to these disorders would have increased risk of preeclampsia in a case/control sample of data from the largest known US preeclampsia GWAS. The authors' findings that; a) risk alleles for raised diastolic blood pressure and increased BMI were strongly associated with preeclampsia risk (more so for the early-onset disease variant), b) risk alleles for raised alkaline phosphatase levels, increased HDL, GFR, and venous thromboembolism were protective, and c) no significant associations for the other traits examined, are consistent with the current status of preeclampsia and HELLP as highly complex disorders with variable clinical presentations depending on pre-pregnancy maternal conditions, fetal/placental genotypes, and maternal adaptation to the challenge of pregnancy. 
So, preeclampsia is far from a Mendelian inherited type of genetic disease and the search for its cause and cure continues 90 years after DeLee's vision!

Conflict of interest: None. A completed disclosure of interest form is available to view online as supporting information. 